\title{
Antimicrobial Resistance Result
}

National Cancer Institute

\section{Source}

National Cancer Institute. Antimicrobial Resistance Result. NCI Thesaurus. Code C85562.

A microbial susceptibility test result in which the growth of microorganisms is not

inhibited by the usually achievable concentrations of antimicrobial agent with normal dosage, or microorganisms demonstrate minimum inhibitory concentrations (MIC) or zone diameters that fall in the range where specific microbial resistance mechanisms are likely in effect, or clinical efficacy of the agent against the microorganism has not been reliably shown in treatment studies. 